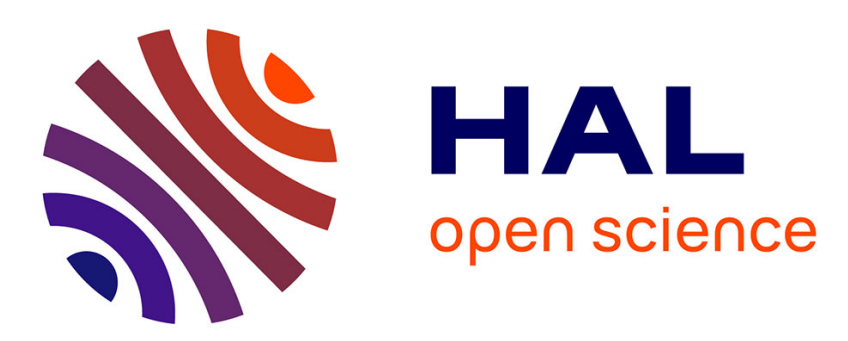

\title{
Achieving High Performance on Supercomputers with a Sequential Task-based Programming Model
}

\author{
Emmanuel Agullo, Olivier Aumage, Mathieu Faverge, Nathalie Furmento, \\ Florent Pruvost, Marc Sergent, Samuel Thibault
}

\section{- To cite this version:}

Emmanuel Agullo, Olivier Aumage, Mathieu Faverge, Nathalie Furmento, Florent Pruvost, et al.. Achieving High Performance on Supercomputers with a Sequential Task-based Programming Model. IEEE Transactions on Parallel and Distributed Systems, inPress, 10.1109/TPDS.2017.2766064 hal01618526

\author{
HAL Id: hal-01618526 \\ https://hal.inria.fr/hal-01618526
}

Submitted on 18 Oct 2017

HAL is a multi-disciplinary open access archive for the deposit and dissemination of scientific research documents, whether they are published or not. The documents may come from teaching and research institutions in France or abroad, or from public or private research centers.
L'archive ouverte pluridisciplinaire HAL, est destinée au dépôt et à la diffusion de documents scientifiques de niveau recherche, publiés ou non, émanant des établissements d'enseignement et de recherche français ou étrangers, des laboratoires publics ou privés. 


\title{
Achieving High Performance on Supercomputers with a Sequential Task-based Programming Model
}

\author{
Emmanuel Agullo, Olivier Aumage, Mathieu Faverge, Nathalie Furmento, \\ Florent Pruvost, Marc Sergent and Samuel Thibault
}

\begin{abstract}
The emergence of accelerators as standard computing resources on supercomputers and the subsequent architectural complexity increase revived the need for high-level parallel programming paradigms. Sequential task-based programming model has been shown to efficiently meet this challenge on a single multicore node possibly enhanced with accelerators, which motivated its support in the OpenMP 4.0 standard. In this paper, we show that this paradigm can also be employed to achieve high performance on modern supercomputers composed of multiple such nodes, with extremely limited changes in the user code. To prove this claim, we have extended the StarPU runtime system with an advanced inter-node data management layer that supports this model by posting communications automatically. We illustrate our discussion with the task-based tile Cholesky algorithm that we implemented on top of this new runtime system layer. We show that it enables very high productivity while achieving a performance competitive with both the pure Message Passing Interface (MPI)-based ScaLAPACK Cholesky reference implementation and the DPLASMA Cholesky code, which implements another (non-sequential) task-based programming paradigm.
\end{abstract}

Index Terms-runtime system, sequential task flow, task-based programming, heterogeneous computing, distributed computing, multicore, GPU, Cholesky factorization

\section{INTRODUCTION}

W HILE low-level designs have long been key for delivering reference high performance scientific codes, the ever growing hardware complexity of modern supercomputers led the High Performance Computing (HPC) community to consider high-level programming paradigms as solid alternatives for handling modern platforms. Because of the high level of productivity it delivers, the Sequential Task Flow (STF) paradigm - further introduced in Section 2.1 is certainly one of the most popular of these candidates. Many studies have indeed shown that task-based numerical algorithms could compete against or even surpass state-ofthe-art highly optimized low-level peers in areas as diverse as dense linear algebra [1]-[4], sparse linear algebra [5]-[7], fast multipole methods [8], [9], $\mathcal{H}$-matrix computation [10] or stencil computation [11], [12], to name a few. Moreover, various task-based runtime systems making use of this paradigm ( [3], [13]-[17] to cite a few) have reached a high level of robustness, incurring very limited management overhead while enabling a high level of expressiveness as further discussed in Section 2.2.

The consequence is twofold. First, new scientific libraries based on the STF paradigm and relying on runtime systems have emerged. We may for instance cite the PLASMA [1], MAGMA [2], FLAME [3] and Chameleon [4] dense linear al-

- M. Faverge is with the Bordeaux Institute of Technology.

- S. Thibault is with the University of Bordeaux.

- N. Furmento is with the CNRS.

- M. Sergent is with the CEA/CESTA.

- E. Agullo, O. Aumage, M. Faverge, N. Furmento, F. Pruvost, M. Sergent, and S. Thibault are with Inria and LaBRI.

E-mail: firstname.lastname@inria.fr

August 2, 2017 revision gebra libraries, the PaStiX [5] and QR_Mumps [6] sparse direct solvers, the Fast Multipole Method (FMM) ScalFMM [9] libraries. Second, the OpenMP Architecture Review Board has introduced constructs for independent tasks in the 3.0 and 3.1 revisions and dependent tasks in the 4.0 revision, which is a decisive step towards the standardization of the STF paradigm.

The aforementioned results showed the success of STF in exploiting a complex, modern, possibly heterogeneous node. However, because the STF runtime must maintain a consistent view of the progress, the model has not been so far considered as a scalable candidate for exploiting an entire supercomputer. In the present article, we show that clever pruning techniques (Section 4.3.1) allow us to alleviate bottlenecks without penalizing the gain of productivity provided by the paradigm. Together with a careful design of communication (Section 4.3.2), allocation (Section 4.3.3) and submission (Section 4.3.4) policies, we show that this approach makes the STF model extremely competitive against both the native MPI and the Parameterized Task Graph (PTG) paradigms.

We carefully present the impact on performance and on the compactness of the different paradigms considered in this study. We illustrate our discussion with the tile Cholesky factorization algorithm [1], [18] from the Chameleon solver [4] running on top of the StarPU runtime system [19]. The Cholesky algorithm aims at factorizing Symmetric Positive Definite (SPD) matrices. We chose to illustrate our discussion with this routine as it is a simple algorithm (composed of only three nested loops as shown in Algorithm 1), and yet it is the reference factorization routine used in state-of-the-art libraries such as FLAME, 


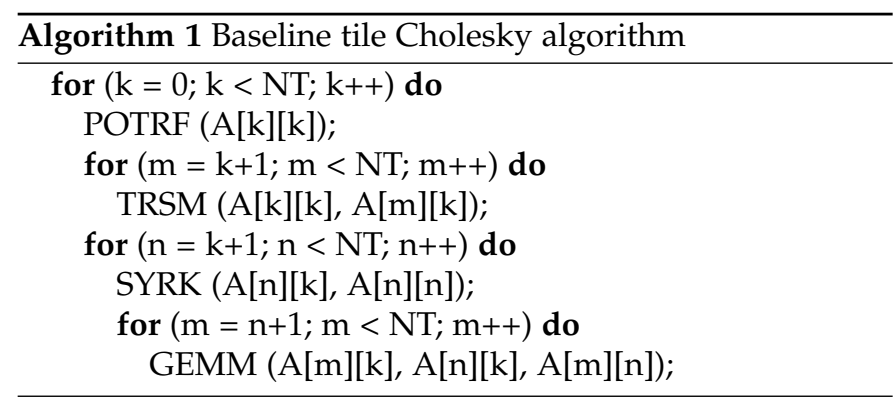

LAPACK, ScaLAPACK, PLASMA, DPLASMA, or MAGMA for solving linear systems involving SPD matrices. Tile algorithms [1], first implemented in the PLASMA library for multicore architectures, are now reference algorithms on parallel architectures and have been incorporated into the Chameleon, FLAME, Intel MKL and DPLASMA libraries. While PLASMA relies on the light-weight Quark [15] runtime system, Chameleon is a runtime-oblivious extension of PLASMA designed for algorithm research that can run on top of many runtime systems, including Quark and StarPU. Chameleon enables one to write algorithms in a way very close to Algorithm 1, thus making it very effective, thanks to the STF model. The MORSE (Matrices Over Runtime Systems at Exascale) project collects such STF-based frameworks and proposes also sparse linear algebra, fast multipole methods, conjugate gradient, etc.

In summary, previous work has shown the potential of the STF model on single-node heterogenous systems, both for ease of programming and obtained performance. The contribution of this article is to extend it to MPI clusters and study its potential for running at large scale on a parallel distributed supercomputer. This includes how the STF model can be safely extended to distributed memory, by generating the needed MPI communications automatically, and a few optimizations which are necessary for scalability. We chose to extend the StarPU (read *PU) runtime system to illustrate this in order to inherit from its ability to abstract the hardware architecture (CPU, GPU, ... ), hence being able to exploit heterogeneous supercomputers.

The rest of the paper is organized as follows. Section 2 presents task-based programming models (Section 2.1), runtime systems that support them in a distributed memory context (Section 2.2) and the baseline (non-distributed) version of the StarPU runtime system (Section 2.3). Section 2.4 presents an extension of StarPU to support explicit MPI calls for exploiting distributed memory machines [20]. Section 3 builds on top of this extension to make communication requests implicit (first contribution), i.e. transparent to the programmer and automatically posted by the runtime system, provided that an initial data mapping is supplied by the programmer. Section 4 presents a detailed performance analysis together with the list of optimizations needed for efficiently supporting the STF model on modern supercomputers (second contribution) before concluding remarks are discussed in Section 5.

\section{BACKGROUND}

\subsection{Task-based programming models}

Modern task-based runtime systems aim at abstracting the low-level details of the hardware architecture and enhance the portability of performance of the code designed on top of them. In many cases, this abstraction relies on a directed acyclic graph (DAG) of tasks. In this DAG, vertices represent the tasks to be executed, while edges represent the dependencies between them.

Each runtime system usually comes with its own API which includes one or multiple ways to encode the dependencies and their exhaustive listing would be out of the scope of this paper. However, we may consider that there are two main modes for encoding dependencies. The most natural method consists in declaring explicit dependencies between tasks. In spite of the simplicity of the concept, this approach may have limited productivity in practice as some algorithms have dependencies that are cumbersome to express. Alternatively, dependencies originating from tasks accessing and modifying data may be implicitly computed by the runtime system thanks to sequential consistency. In this latter approach, tasks are submitted in sequence and data they operate on are also declared.

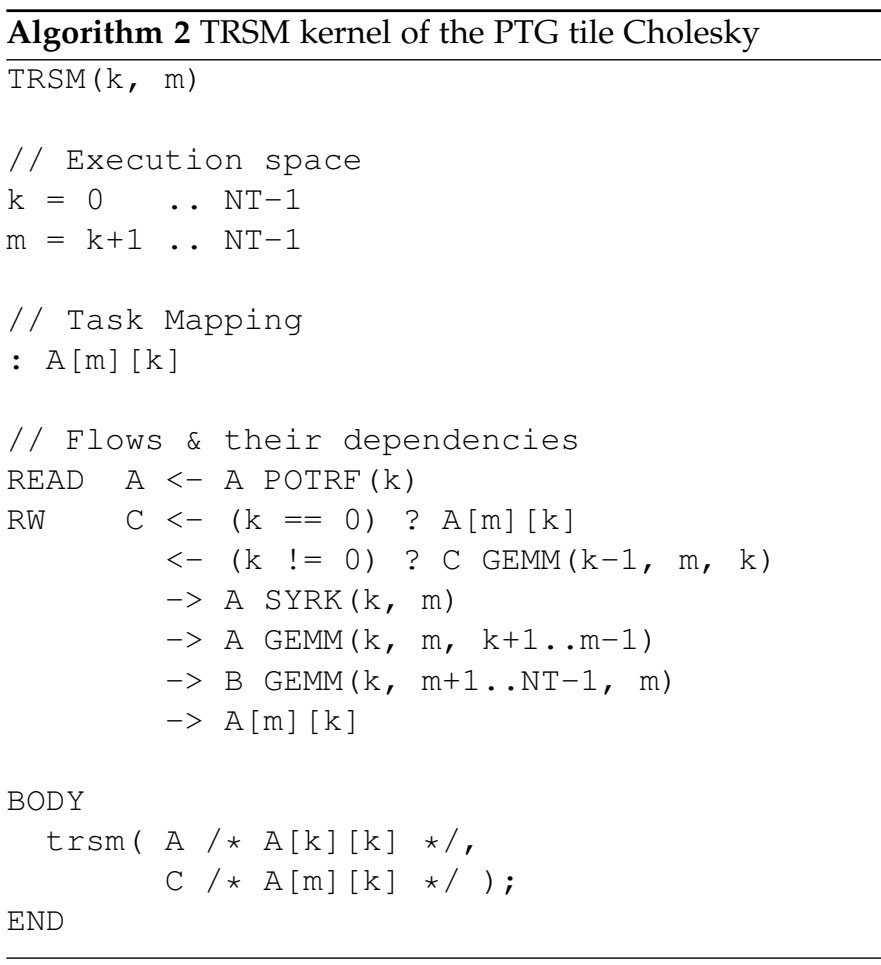

Depending on the context, the programmer affinity and the portion of the algorithm to encode, different paradigms may be considered as natural and appropriate and runtime systems often allow them to be combined. Alternatively, one may rely on a well-defined, simple, uncluttered programming model in order to design a simpler, easier to maintain code and to benefit from properties provided by the model.

One of the main task-based programming model relying on explicit dependencies between tasks is certainly the Parameterized Task Graph (PTG) model [21]. In this model, tasks are not enumerated but parameterized and dependencies between tasks are explicit. For instance, Algorithm 2 shows 
how the TRSM task from the above tile Cholesky algorithm is encoded in this model. For conciseness, we do not show the POTRF, SYRK and GEMM pseudo-codes here; they are similar and further details can be found in [22]. The task is parameterized with $k$ and $m$ indices. Its execution space is defined as the range of values that these indices can have (for instance $k$ varies from 0 to $N T-1$ ). Assuming that a data mapping has been defined separately and ahead of time (for all tiles and hence for tile $A[m][k]$ in particular), a task mapping is provided by assigning task $\operatorname{TRSM}(k, m)$ onto the resource associated with a certain data $(A[m][k]$ here). Then, dependencies are explicitly stated. For instance, $\operatorname{TRSM}(k, m)$ depends on POTRF $(k)$ (left arrow) and, conversely, $S Y R K(k, m)$ may depend on it (right arrow). Correctly expressing all the dependencies is not trivial, as one can see in the pseudo-code. Furthermore, in order to handle distributed memory machines, the data-flow must also be provided. TRSM has to retrieve two data, referenced as $A$ and $C$ in its body (where the actual code must be provided). In the particular case of the dependency between $\operatorname{TRSM}(k, m)$ and $\operatorname{POTRF}(k)$, POTRF produces one single data (noted $A$ in the pseudo-code of POTRF), which must be transferred to TRSM and referenced as $A$ for future usage within the body. This encoding of the DAG induces a low memory footprint and ensures limited complexity for parsing it while the problem size grows. Furthermore, since dependencies are explicit, the DAG can be naturally unrolled concurrently on different processes in a parallel distributed context, a key attribute for achieving scalability.

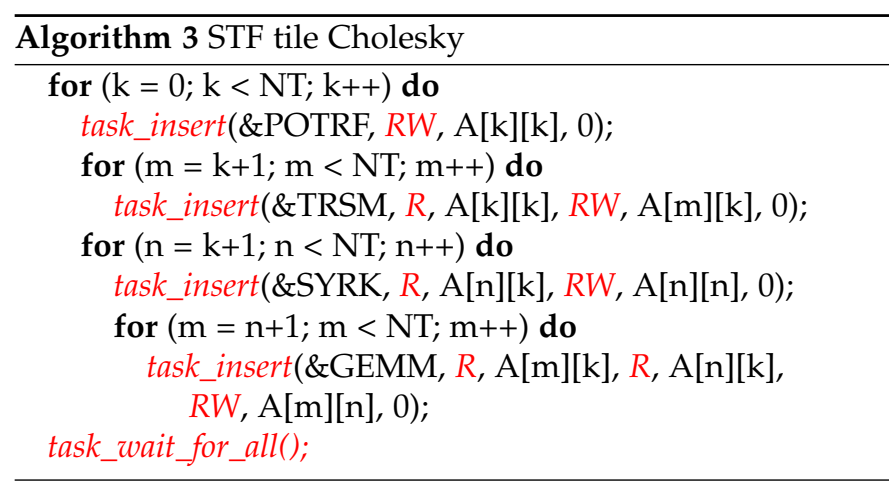

On the other hand, the Sequential Task Flow (STF) programming model consists in fully relying on sequential consistency, exclusively using implicit dependencies. This model enables the programmer to write a parallel code that preserves the structure of the sequential algorithm, enabling very high productivity. Indeed, writing an STF code consists of inserting a sequence of tasks through a non-blocking function call (which we call "task_insert") that delegates the asynchronous, parallel execution of the tasks to the runtime system. Upon submission, the runtime system adds a task to the current DAG along with its dependencies which are automatically computed through data dependency analysis [23]. The task becomes ready for execution only when all its dependencies are satisfied. A scheduler is then in charge of mapping the execution of ready tasks to the available computational units. In this model, the sequential tile Cholesky algorithm can simply be ported to the STF model as proposed in Algorithm 3. The only differences with the original Algorithm 1 are the use of task_insert instead of direct function calls, and the addition of explicit data access mode ( $\mathrm{R}$ or $\mathrm{RW}$ ). The increasing importance of this programming model in the last few years led the OpenMP board to extend the standard to support the model through the task and depend clauses in the revision 4.0. This paradigm is also sometimes referred to as superscalar since it mimics the functioning of superscalar processors, where instructions are issued sequentially from a single stream but can actually be executed in a different order and, possibly, in parallel depending on their mutual dependencies.

In a parallel distributed context, maintaining sequential consistency requires the runtime to maintain a global view of the graph. In the literature, different approaches have been proposed. ClusterSs [13] has a master-slave model where a master process is responsible for maintaining this global view and delegates actual numerical work to other processes. The authors showed that the extreme centralization of this model prevented it from achieving high performance at scale. On the contrary, in the extension of Quark [24], all processes fully unroll the DAG concurrently. Although requiring fewer synchronizations, the authors showed that the approach was also limited at scale, as each process is still required to fully unroll the DAG, and in spite of the lower number of synchronizations, that symbolic operation is performed redundantly and takes a significant amount of time, possibly higher than the time spent for numerical computations. In the present article, we study the potential of the STF paradigm for programming modern supercomputers relying on the StarPU runtime system (see Section 2.3). As in [24], all processes fully unroll the DAG concurrently. However, while the full unrolling of the DAG was a serious drawback for performance in Quark, we propose here to alleviate this bottleneck by performing a clever pruning of the DAG traversal (see Section 4.3.1) to entirely eliminate irrelevant dependence edge instantiation. We show that the STF model then becomes competitive against both the native MPI and PTG paradigms.

\subsection{Short review of task-based runtime systems for distributed memory platforms}

Several related approaches have been proposed within the community. StarSs is a suite of runtime systems developed at the Barcelona Supercomputing Center and supporting the STF model. Among them, ClusterSs [13] provides an STF support for parallel distributed memory machines with a master-slave interaction scheme. OmpSs [14] targets SMP, SMP-NUMA, GPU and cluster platforms. Master-slave schemes may suffer from scalability issues on large clusters due to the bottleneck constituted by the master, though OmpSs supports task nesting on cluster nodes, to reduce the pressure on the main master node. On the contrary, the extension of Quark for distributed memory machines proposed by YarKhan [24] relies on a decentralized approach to support the STF model. The present study extends this decentralized approach with the StarPU runtime system.

The PaRSEC runtime system [2], [25] developed at UTK, supports the PTG model (as well as other Domain Specific Languages). Dependencies between tasks and data are expressed using a domain specific language named JDF and compiled into a compact, parametric representation of 
the dependence graph. The size of the PTG representation does not depend on the size of the problem, but only on the number of different tasks used by the application, allowing for an efficient and highly scalable execution on large heterogeneous clusters. JDF also ensures support for irregular applications. Because dependencies are explicitly provided by the application, the model is extremely scalable: the runtime can explore the DAG from any local task to any neighbor task. PaRSEC exploits this property to ensure excellent scalability. The DPLASMA library is the dense linear algebra library implemented on top of PaRSEC that originally motivated the design of this runtime. It is highly optimized and can be viewed as a reference implementation of a library on top of PaRSEC (although many other scientific codes have been implemented on top of it since then). DPLASMA/PaRSEC was used as a reference code in [24] to assess the limits of the STF support for distributed memory platforms in the proposed extension of Quark. It will also be our reference code.

The SuperGlue [26] environment, developed at Uppsala University, provides a model based on data versioning instead of using a DAG of tasks. SuperGlue only implements a single scheduling algorithm: locality-aware work stealing. It is limited to single node, homogeneous multicore platforms. However it can be associated with the DuctTEiP [16] environment, to target homogeneous clusters.

The Legion [17] runtime system and its Regent compiler [27], developed at Stanford University, provide a taskbased environment supporting distributed memory platforms and GPUs, programmable at the Legion library level directly, or at a more abstract level through the Regent dedicated language and compiler. Legion enables the programmer to define logical regions similar to StarPU's registered data, and express dependencies between tasks and logical region accesses. In contrast to StarPU tasks, Legion tasks may spawn child tasks and wait for their completion. StarPU tasks must instead run to completion as a counterpart for enabling accurate performance model building.

The TBLAS environment [28], initially designed at UTK, is a task-based runtime system for clusters equipped with GPUs. It makes use of both the general purpose cores and the GPUs for computations. However, tasks as well as data are distributed to hosts and GPUs statically contrary to the approach we consider in the present paper where any task can run on any computational unit thanks to the abstraction of the STF model we consider.

Many other runtimes have been designed over the years. APC+ [29] (UNC Charlotte) and Qilin [30] (Georgia Tech.) only optimize scheduling for a single kind of computing kernel per application. ParalleX/HPX [11] (LSU) supports both parallel and distributed memory platforms, but no detail is given about accelerator support. Most of them perform reactive/corrective load balancing using approaches such as work stealing [31] (Inria) or active monitoring [32], [33] (Univ. of Illinois) while StarPU, used for this work, attempts to proactively map computations in a balanced manner using performance models.

PGAS languages such as UPC (UPC Consortium) or XcalableMP (XMP Specification Work. Group) provide distributed shared memory and are being extended to support NVIDIA CUDA devices [34], [35]. The proposed interfaces are however mostly guided by the application, without dynamic scheduling, thus leaving most of the load balancing burden on the application programmer.

\subsection{StarPU: a task-based runtime system for heteroge- neous architectures}

The basis for this work is the StarPU [19] runtime system, which deals with executing task graphs on a single heterogeneous node, composed of both regular CPU cores and accelerators such as GPUs.

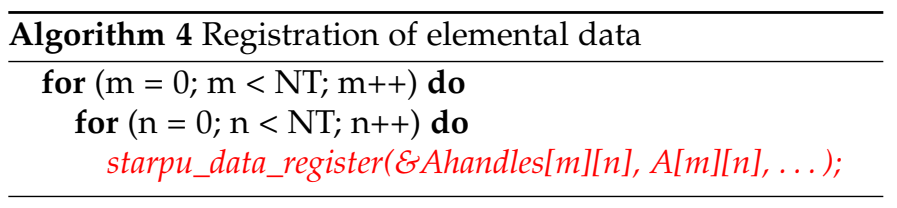

The first principle of StarPU is that the application first registers its data buffers to StarPU, to get one handle per data buffer, which will be used to designate this data, as shown in Algorithm 4. This enables StarPU to freely transfer the content of those handles back and forth between accelerators and the main memory when it sees fit, without intervention from the application. The second principle is that for each operation to be performed by tasks (the GEMM, GEneral Matrix Multiplication, for instance), a codelet is defined to gather the various implementations: the DGEMM CPU implementation from MKL and the cublasDgemm GPU implementation from CUBLAS, for instance. A task is then simply a codelet applied on some handles. The StarPU runtime system can then freely choose when and on which CPU core or accelerator to execute the task, as well as when to trigger the required data transfers. For instance, the Chameleon framework provides codelets for the classical dense linear algebra kernels.

As a consequence, StarPU can optimize task scheduling by using state-of-the-art heuristics. Estimations of task completion time can typically be obtained by measuring them at runtime [36], or can be provided explicitly by the application. Thus, StarPU provides multiple scheduling heuristics ranging from basic strategies, such as eager or work stealing, to advanced strategies such as HEFT [37]. These can take into account both computation time and CPU-Accelerator device data transfer time to optimize the execution.

StarPU can further optimize these data transfers between the main memory and the accelerators' memory. Since data registration makes StarPU responsible for managing data buffers locations, StarPU can keep track of which of the main memory and/or accelerators' memory have a valid copy of a given data, using its distributed shared-memory manager. For instance, this makes it possible to replicate data over all accelerators which need them. By making scheduling decisions in advance, StarPU can also start the required data transfers early, thus overlapping them with the currently running tasks. StarPU can also keep data in an accelerator as long as it is used repeatedly by tasks scheduled on it, thus avoiding duplicate transfers. On the contrary, when room must be made in the accelerator memory, it can also proactively evict unused data.

StarPU supports the STF model. The tile algorithm proposed in Algorithm 3 can be executed with StarPU, by 


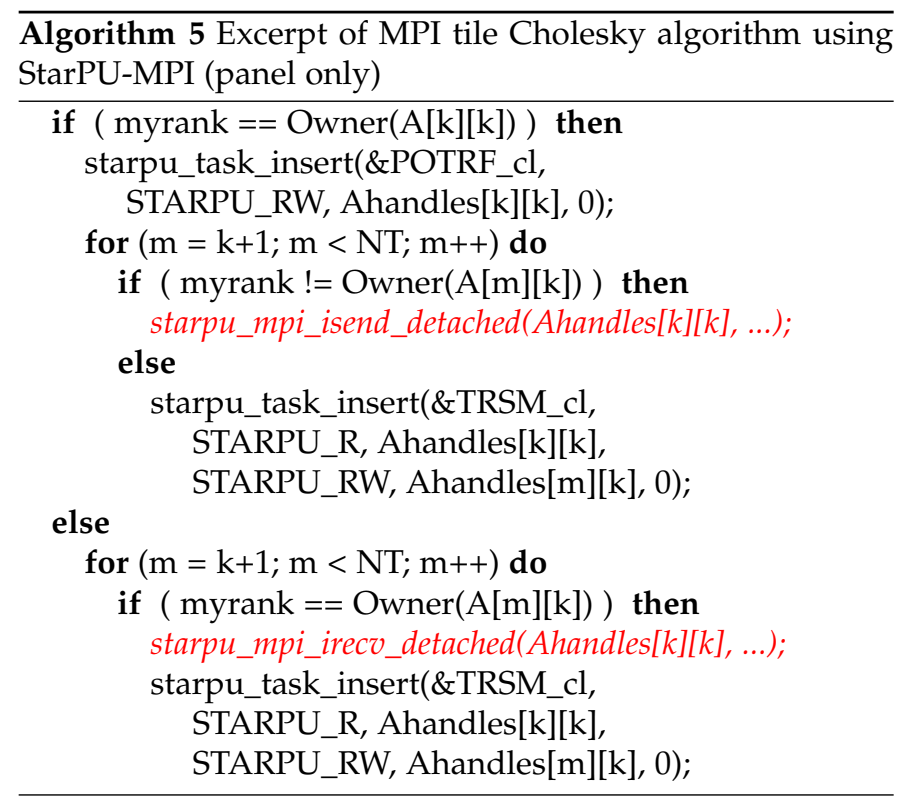

simply replacing the task_insert call by starpu_task_insert, and the task_wait_for_all call by starpu_task_wait_for_all.

\subsection{StarPU-MPI: Explicit message passing support for StarPU}

While the StarPU runtime system [19] was intended to support the execution of a task-based code on a single node, we now present a mechanism proposed in [20], which handles distributed memory platforms, named StarPU-MPI in the sequel. Provided the huge amount of existing MPI applications, one may indeed want to make it possible to accelerate these so that they can take full advantage of accelerators thanks to StarPU, while keeping the existing MPI support as it is. Instead of having a single instance of StarPU distributed over the entire cluster, the approach runs an instance of StarPU on each MPI node and lets them communicate with each other through StarPU-MPI. The flexibility of this hybrid programming model has also been illustrated in the case of MPI applications which call libraries written in OpenMP or TBB for instance.

Algorithm 5 shows how to write the tile Cholesky algorithm with this paradigm. For conciseness and clarity, we focus on the panel factorization only (a POTRF kernel and the subsequent TRSM kernels) and present a non-optimized version. All function calls are asynchronous and allow for overlapping communications with task executions. The for loops thus complete quickly (they only submit requests to StarPU and StarPU-MPI) and the only synchronization point possibly occurs at the end of the application at the starpu_task_wait_for_all step, which ensures the completion of all submitted tasks. The detached calls perform asynchronous data transfers: for instance, the send requests will actually be posted to MPI only when the tasks which produce the piece of data are finished, and tasks which depend on data coming from a reception request will also wait for the reception to complete.

While we presented a non-optimized version of the code and restricted ourselves to the panel factorization only, one can observe that the code already differs from the
STF paradigm. In the case of more complex algorithms (including a full and optimized version of the tile Cholesky factorization), this model may lead to error-prone and hard to maintain codes. In the sequel, we propose a programming model that sticks as much as possible to the STF paradigm by posting communications automatically. This enables programmers to exploit an entire modern supercomputer without suffering from the complexity of such a hybrid programming model.

\section{SEQUENTIAL TASK FLOW WITH IMPLICIT MES- SAGE PASSING}

Just like explicit dependencies, explicitly specifying MPI communications is tedious and error-prone. We now propose to automatically infer those communications in order to maintain a compact STF code while exploiting a full heterogeneous supercomputer. The principle is the following. An initial data mapping over the MPI nodes is supplied by the application, and the sequence of tasks is identically submitted by the application on all MPI nodes. Each node can then unroll the whole application task graph, and automatically determines by itself which subset of tasks it should run (according to the data mapping), which MPI transfers it should initiate to resolve an out-going internode dependence edge, and which incoming MPI transfers it should expect resulting from its own inbound internode dependence edges. Indeed, an MPI send has to be automatically initiated when local data is needed by a task running on an other MPI node, and an MPI receive has to be initiated when a local task needs a data which is mapped on another MPI node. Put another way, an MPI transfer is considered for each task graph edge between two tasks which are to be executed on different MPI nodes. Subsequently, no coherency synchronization is needed between nodes by construction, beyond the necessary user data transfers. Figure 1 illustrates those data transfers. A cache mechanism, described in Section 4.3.2, avoids duplicate communications. Moreover, since unrolling the whole task graph on each node can become costly at scale, we discuss in Section 4.3.1 how we refine the model to overcome this.

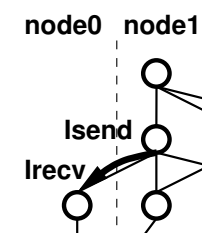

Fig. 1. Example of communication inferred from task graph edge.

\subsection{Data mapping}

The data mapping determines which MPI node owns which data, that is for each data, the node responsible for keeping the last value and for sending it to MPI nodes which need it. The initial data mapping, as specified by the application, can remain static or be altered by the application throughout the execution. For dense linear algebra, the two-dimensional block-cyclic data distribution [38] provides a good example of static mapping, shown in Algorithm 6. 


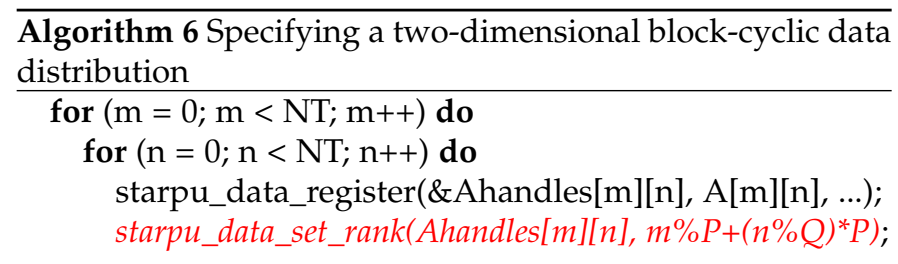

When the data mapping is altered by the application, the new data mapping information must be submitted on every node at the same logical point in the sequential task submission flow. The model indeed requires all nodes to have the same knowledge of the data distribution, for Sends and Receives to be posted coherently. However, the data mapping information is only used at submission time, to determine which MPI send and receive requests to initiate to resolve dependencies arising from tasks being submitted. Thus, such a redistribution step is not a problem for performance: computing a new data redistribution can be done asynchronously while the previously submitted tasks are still being scheduled and executed. Once determined, the new data redistribution is enforced asynchronously and transparently, by initiating MPI transfers in the background. Meanwhile, subsequent task submissions now proceed using the new data distribution.

This allows the runtime to overlap redistribution with computation: a part of the graph is submitted, some of it is executed, statistics can be collected on it, a new data distribution can be computed accordingly while the submitted tasks continue executing, and another part of the graph can be submitted with the new distribution, etc. Provided that the time to compute the new distribution is smaller than the time to execute the part of the task graph that is already submitted and not used for collecting statistics, the execution of the application will never be stalled. For very large systems, a global redistribution would be very costly, possibly beyond the time to process the already-submitted tasks. Automatic local redistribution schemes could be used instead in this case and are being considered as future work within StarPU, to relieve the application from having to implement it.

\subsection{Task mapping}

The task mapping can be made static too, as specified by the application. However, it is usually more convenient to let it be automatically determined by StarPU from the data mapping, that is, let tasks move to data. By default, StarPU will execute a task on the node which owns the piece of data to be written to by that task. Other cheap automatic heuristics are available in StarPU, or can be provided by the application to avoid having to specify both a data mapping and a task mapping.

\subsection{Discussion}

All in all, by inferring the MPI transfers automatically from data dependence edges, separating the data distribution from the algorithm, and inferring task mapping automatically from the data distribution, the distributed version of tile Cholesky boils down to Algorithm 7. The only difference with Algorithm 3 in the main loop is the usage of the starpu_mpi_task_insert directive instead of starpu_task_insert. This function call determines if communications have to be posted through the StarPU-MPI layer (described in Section 2.4) additionally to the baseline StarPU actual computational task submission. In the context of the Chameleon framework, all these details are actually hidden behind function calls which look very much like BLAS calls, and the eventual implementation is actually very close to the original Algorithm 1. The only addition is the optional starpu_mpi_cache_flush calls, which can be ignored for the moment. We will motivate their usage in Section 4.3.2.

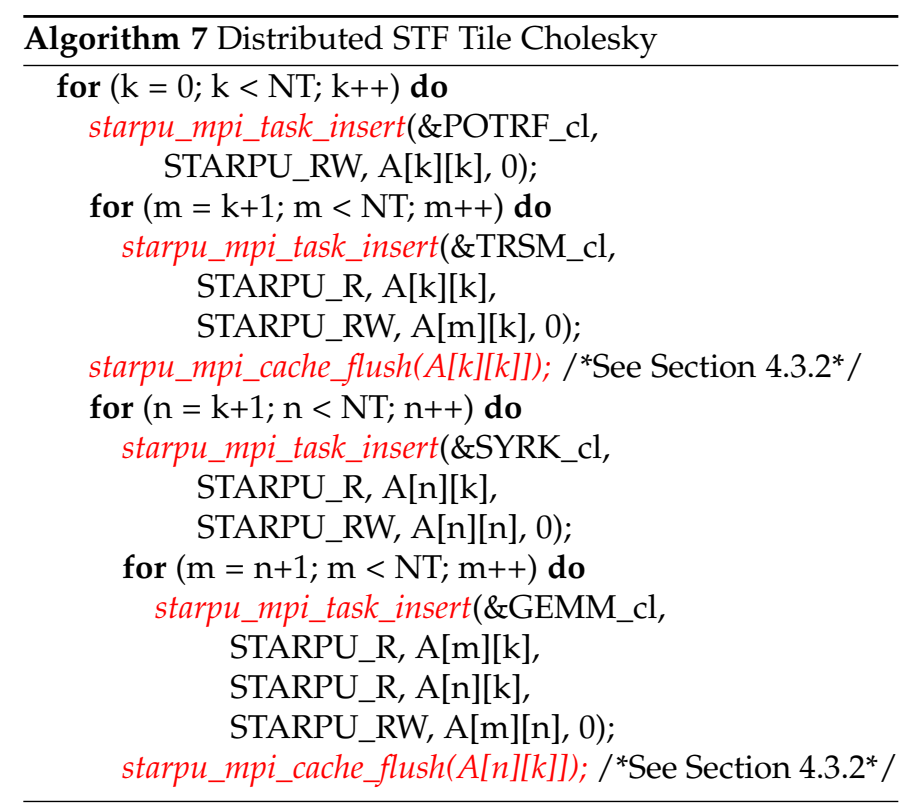

The overall algorithm, sequential and extremely compact, hence ensures very productive development and maintenance processes: algorithms can be designed and debugged within a sequential context, and parallelism can be safely enabled afterwards with the runtime system, transparently for the programmer.

\section{EXPERIMENTS}

As discussed above, the STF model removes the programming burden of explicitly posting the communications (required by the MPI paradigm) or explicitly providing the dependencies (required by the PTG paradigm) as it infers them automatically from the data mapping. We now show that it is possible to rely on this sequential task-based paradigm while achieving high performance on supercomputers. After describing the experimental context (Section 4.1), we first present the final results we obtained (Section 4.2). We eventually list the major issues we faced together with the solutions we have devised to cope with them (Section 4.3) in order to ensure an overall extremely efficient support for the STF model on modern distributed memory machines. We focused on the particular case of a dense linear algebra algorithm to illustrate our study because DPLASMA/PaRSEC is a solid reference in terms of scalability of high performance numerical libraries and was also the reference code in [24]. 


\subsection{Experimental Context}

We implemented the tile Cholesky factorization proposed in Algorithm 7 within the Chameleon library, extending [4] to handle distributed memory machines. We compare the resulting code with two state-of-the-art distributed linear algebra libraries: DPLASMA and ScaLAPACK. DPLASMA implements a highly optimized PTG version of the tile Cholesky factorization introduced in Algorithm 2 on top of the PaRSEC runtime system. ScaLAPACK is the MPI state-of-the-art reference dense linear algebra library for homogeneous clusters. All three libraries rely on the same two-dimensional block-cyclic data distribution [38] between MPI nodes, and thus exhibit the same availability of parallelism between MPI nodes and communication requirements, to make the comparison fair. In practice, DPLASMA and Chameleon library achieve the same communication overlap through the runtime system, thus not suffering from communication latencies, as opposed to the standard ScaLAPACK implementation. All the experiments were conducted in real double precision arithmetic.

We conducted our experiments on two platforms. On the one hand, we experimented on up to 144 heterogeneous nodes of the TERA-100 heterogeneous cluster [39] located at CEA, France. The architectural setup of the nodes and the libraries we used is as follows:

- CPU QuadCore Intel Xeon E5620 @ 2.4 GHz x 2,

- GPU NVIDIA Tesla M2090 (6 GiB) x 2,

- $24 \mathrm{GiB}$ main memory,

- Infiniband QDR@ $40 \mathrm{~Gb} / \mathrm{s}$,

- BullxMPI 1.2.8.2,

- Intel MKL 14.0.3.174,

- NVIDIA CUDA 4.2.

On the other hand, we experimented on up to 256 homogeneous nodes of the Occigen cluster [40] located at CINES, France. The setup is as follows:

- CPU 12-cores Intel Xeon E5-2690 @ 2.6 GHz x 2,

- $64 \mathrm{GiB}$ main memory,

- Infiniband 4x FDR @ 56 Gb/s,

- BullxMPI 1.2.8.4-mxm,

- Intel MKL 17.0.

For our experiments, we distinguish two setups depending on whether GPUs are used for computation or not. The former will be called the heterogeneous setup, while the latter will be called the homogeneous setup. We tuned the block size used by each linear algebra library and the schedulers used by each runtime system for each setup in order to get the best asymptotic performance for each library. Table 1 shows the resulting set up on both machines. In the homogeneous case this tuning procedure led to a larger tile size (512) on the Occigen cluster for both codes. In the heterogeneous case, note that DPLASMA is able to efficiently exploit a lower tile size (320) than Chameleon (512) as it supports multi-streaming (see below). PaRSEC and StarPU respectively rely on their own Priority Based Queue $(P B Q)$ and priority (prio) schedulers, which are both hierarchical queuebased priority scheduler implementations. In the heterogeneous case, StarPU relies on the dmdas scheduler, a dynamic variant of the HEFT algorithm, and PaRSEC relies on an extension of PBQ allowing for greedy GPU offloading [41].
Note that StarPU schedulers have been mostly optimized for heterogeneous architectures and do not efficiently take into account locality effects (cache, NUMA) of the CPU memory. This is not significant for a heterogeneous machine whose performance is mostly driven by GPU performance, but noticeably impacts modern homogeneous multicore machines. For instance, a parallel GEMM executed with prio on a single node of the Occigen computer achieves only $82 \%$ of the GEMM theoretical node peak (computed as the sum of the best GEMM CPU single core over all matrix sizes over all 24 cores of the node).

TABLE 1

Tuned parameters on the TERA-100 and the Occigen machines. The block size represents the tile size in the Chameleon and DPLASMA cases and the panel width in the ScaLAPACK case.

\begin{tabular}{lcccc}
\hline \multirow{2}{*}{ Model/Library } & \multicolumn{2}{c}{ CPU-only } & \multicolumn{2}{c}{ CPU + GPU } \\
\cline { 2 - 5 } & Block size & Sched. & Block size & Sched. \\
\hline \hline TERA-100 & \multicolumn{5}{c}{} \\
\hline STF/Chameleon & 320 & prio & 512 & dmdas \\
PTG/DPLASMA & 320 & PBQ & 320 & PBQ \\
MPI/ScaLAPACK & 64 & static & - & - \\
\hline \hline Occigen & & & & \\
\hline STF/Chameleon & 512 & prio & - & - \\
PTG/DPLASMA & 512 & PBQ & - & - \\
\hline
\end{tabular}

\subsection{Final results}

We present in this section the final performance results obtained in our study, with all optimizations presented in this paper enabled. Table 2 lists the implementation-specific parameters which were selected during the experiments, to enhance performance to the best of each runtime capability. The main differences are the following:

- While StarPU can execute any kernel of the Cholesky factorization on GPUs, PaRSEC chooses to offload only GEMM kernels on GPUs since this kernel is the most compute intensive one. PaRSEC has support for multi-streaming on GPUs, which makes it possible for several kernels to be executed at the same time on a single GPU.

- Regarding the communications, the STF model supported by StarPU with Algorithm 7 infers point-topoint communications (we discuss in Section 4.3.2 how to alleviate their repetitions). The PTG model implemented by PaRSEC with Algorithm 2 supports collective communication patterns.

- In addition to the memory pinning used by CUDA for CPU-GPU transfers, StarPU takes advantage of the memory pinning optimization of the OpenMPI library to accelerate communications, while it has been turned off with PaRSEC as it induced notable slowdowns.

Figure 2 shows the performance obtained on the TERA100 cluster, both in the homogeneous (only CPUs being used) and the heterogeneous (all computational units being used) cases. Figure 3 shows the performance on the Occigen cluster. In both cases, all the optimizations presented in this 
TABLE 2

Implementation-specific parameters for each runtime system.

\begin{tabular}{lllll}
\hline $\begin{array}{l}\text { Model/Library } \\
\text { (runtime) }\end{array}$ & $\begin{array}{l}\text { Schedulable } \\
\text { kernels on } \\
\text { GPUs }\end{array}$ & $\begin{array}{l}\text { GPU } \\
\text { multi- } \\
\text { streaming }\end{array}$ & $\begin{array}{l}\text { MPI } \\
\text { pomm. } \\
\text { policy }\end{array}$ & $\begin{array}{l}\text { OpenMPI } \\
\text { memory } \\
\text { pinning }\end{array}$ \\
\hline $\begin{array}{l}\text { STF/Chameleon } \\
\text { (StarPU) }\end{array}$ & All & No & $\begin{array}{l}\text { Point-to- } \\
\text { point }\end{array}$ & Yes \\
$\begin{array}{l}\text { PTG/DPLASMA GEMM } \\
\text { (PaRSEC) }\end{array}$ & only & Yes & Collectives & No \\
\hline
\end{tabular}

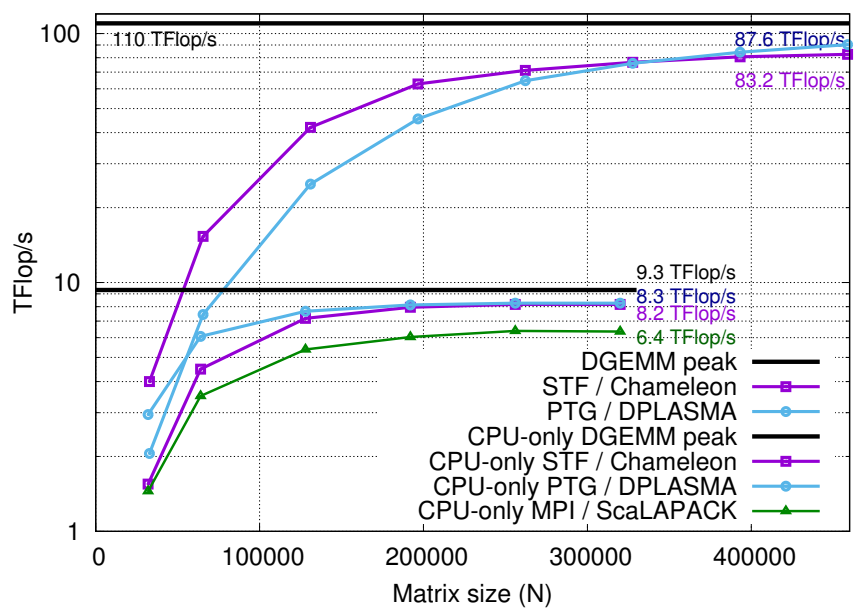

Fig. 2. Final performance results on all 144 nodes of the TERA-100 cluster (1152 CPU cores and 288 GPUs). The Y-axis is a logarithmic scale.

paper were enabled. The main observation is that the STF model is competitive with the PTG and the MPI programming paradigms. This is the main message of this paper: it shows that very high performance can be reached with the high level of productivity delivered by this model. Furthermore, the observed differences in terms of performance between Chameleon (which implements the STF model) and DPLASMA (which implements the PTG model) are mostly due to the inherent low-level features of the underneath runtime systems (StarPU and PaRSEC, respectively) discussed above, and the behavior of the MPI implementation, but not to the respective programming models.

We first discuss the homogeneous setups. On small matrices, DPLASMA slightly outperforms Chameleon on both machines. Indeed, StarPU is optimized for heterogeneous architectures while PaRSEC has been originally designed to efficiently exploit homogeneous clusters. In this particular case, the prio scheduler implemented in StarPU would need to have support for locality as does the PBQ scheduler implemented in PaRSEC. This is thus not related to the programming model itself but to the low-level (yet important) details of the internal design of the respective runtime systems. For large matrices, Chameleon catches up with DPLASMA because there is enough computation to completely overlap data transfers with computation. On the TERA-100 machine, Chameleon achieves $87.5 \%$ of the GEMM theoretical peak on matrices of order $N=320.000$. On the Occigen platform, the achieved performance is however only $72.4 \%$ on matrices of order $N=786.432$.

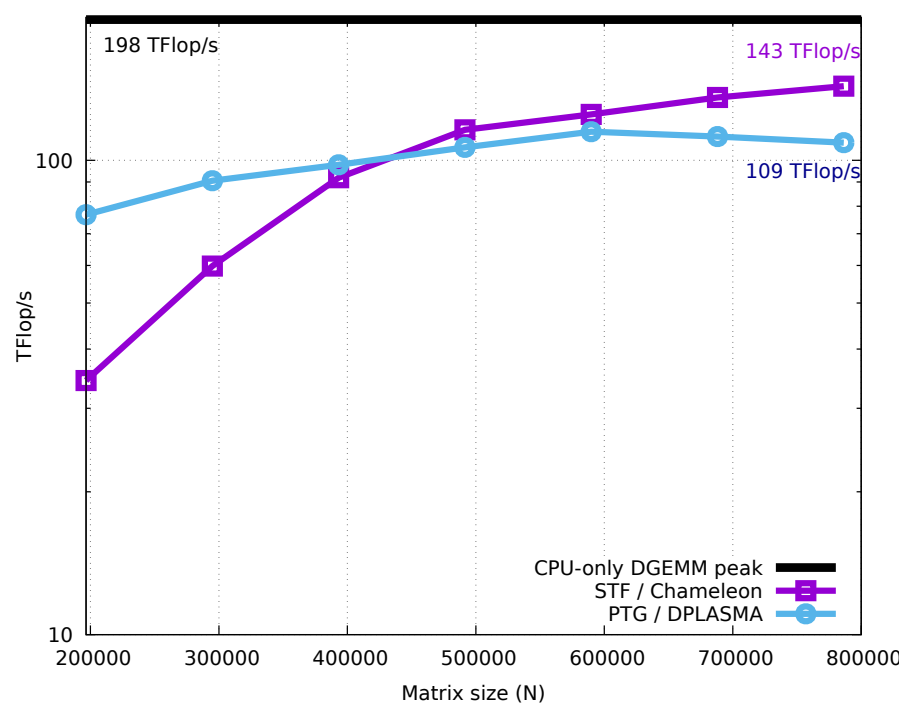

Fig. 3. Final performance results on all 256 nodes of the Occigen cluster (6144 CPU cores).

Indeed, the StarPU prio scheduler is not optimized for such a modern multicore architecture (see discussion in Section 4.1). We will show that the STF model itself (with proper pruning) does not hurt the performance in Section 4.3.1. On the TERA-100 machine, we also compared the task-based approaches (Chameleon and DPLASMA) with the MPI reference (ScaLAPACK). We observe that both Chameleon and DPLASMA surpass ScaLAPACK, showing that task-based codes on top of runtime systems positively compete with an MPI reference code. ScaLAPACK uses a panel-based factorization which simplifies the static scheduling of the computation, but limits the maximum available parallelism.

In the heterogeneous setup, Chameleon outperforms DPLASMA up to matrices of order 320,000. The OpenMPI memory pinning greatly accelerates StarPU point-to-point communications, thus unlocking enough parallelism to feed GPUs with computation. Furthermore, the StarPU HEFT scheduling policy achieves better decisions on the starting phase of the factorization, while the PaRSEC greedy policy enforces all initial updates on the GPU despite the cost of transferring the piece of data. For larger matrices, both StarPU and DPLASMA achieve a performance close to the GEMM peak (computed as the sum of the best GEMM CPU core and GPU performance over all computational units and matrix sizes), with a slight advantage for DPLASMA due to the multi-streaming support of PaRSEC which achieves a better trade-off between parallelism and granularity.

\subsection{List of optimizations required to efficiently support the STF model on supercomputers}

We have shown above that we could successfully achieve competitive performance with the STF programming model on two modern supercomputers against reference linear algebra libraries implemented with the PTG and MPI paradigms. We now present issues we have faced during this study and how we solved them in order to achieve this performance on those distributed memory machines. 
The first issue relates with the STF model inherent requirement, and potential overhead, to submit tasks sequentially for the whole, distributed task graph. We explain in Section 4.3.1 that the characteristics of the global task graph make it suitable for drastic pruning at the node level, thus preserving scalability on large platforms.

The second issue is that inter-node dependence edges trigger duplicate, redundant network data transfers. We discuss in Section 4.3.2 the communication cache mechanism we implemented to filter out redundant network transfers.

The third issue is that the common memory allocator (such as provided by the locally available $\mathrm{C}$ library) incurs either critical overhead or fragmentation. In Section 4.3.3, we study the characteristics of the two memory related system calls provided by Linux and similar operating systems, and how these characteristics impair the $\mathrm{C}$ library memory allocator, and in the end, how StarPU gets impaired as well. We then present our solution to this issue, involving the cooperation of a memory allocation cache mechanism, together with a task submission flow control policy further detailed in Section 4.3.4.

\subsubsection{Pruning of the Task Graph Traversal}

The distributed STF paradigm specifies that the full unrolling of the task graph must be done on all participating nodes, even if only a sub-part of the task graph is actually executed on a given node. Such a requirement could hinder scalability. In reality however, a given node only needs to unroll the incoming, local and outgoing dependence edges from its own point of view (including WAR dependencies). Thus, a node may safely prune a task submission for which it is not an end of any of the task's data dependence edges.

As a result, for each node, testing whether a task must not be pruned reduces to checking whether any data of the task is owned by the node (it will have to supply the piece of data), or whether the piece of data that the task writes to was cached by the node (it will have to discard its cached value). These tests are very lightweight, so they can be added as simple if conditions in front of task submissions of Algorithm 7. Furthermore, since these tests actually depend only on the type of task, they do not need to appear explicitly in Algorithm 7, but can be hidden in a helper function which makes the task submission or not. In the context of Chameleon for instance, such a helper, which includes the pruning test, exists for each BLAS operation, so that the algorithm programer does not have to care about it, and just calls the BLAS helpers in a loop nest which thus looks very similar to Algorithm 1. For instance, the TRSM helper boils down to Algorithm 8 .

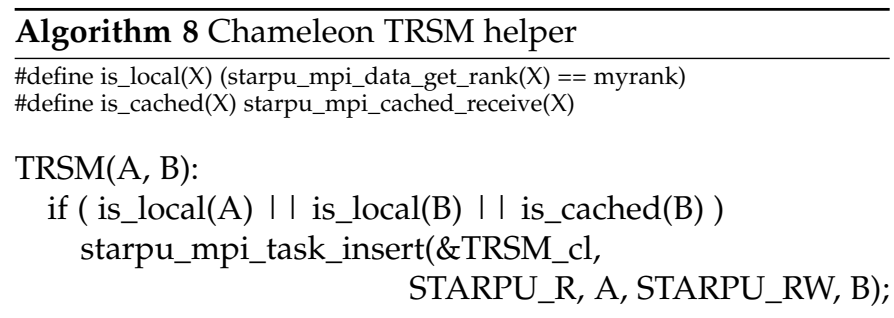

The top of Figure 4 contains a comparative study of task submission time and task execution time depending on
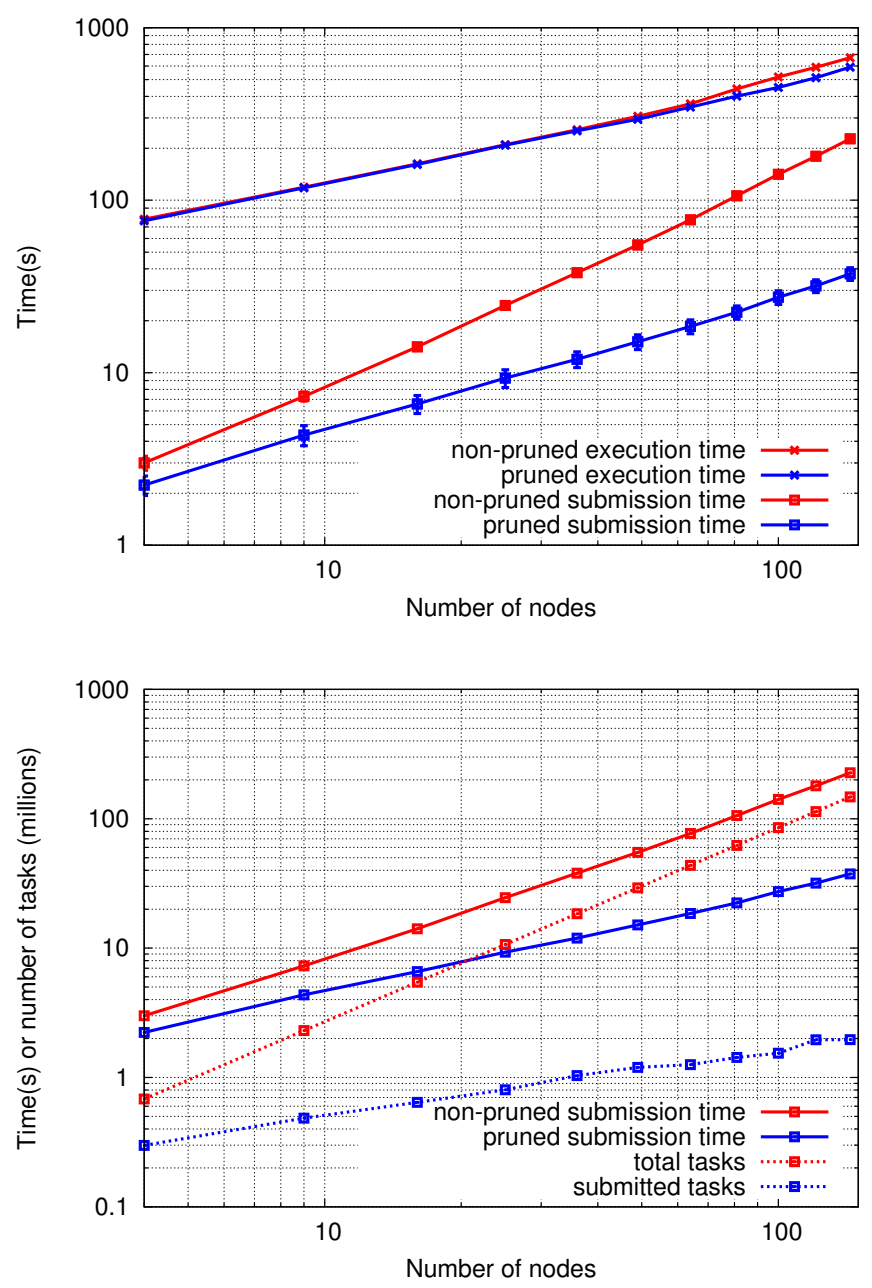

Fig. 4. Impact of pruning the task graph on submission and execution time, and number of submitted tasks. The test case for 1 node is a matrix of size 40,960 , and we keep the same amount of memory per node when increasing the number of nodes.

whether the application pruned the task graph traversal or not by using this simple technique. We first observe that the slope of the execution time without pruning slightly increases beyond 64 nodes, as the resulting of the steep, nonpruned submission time curve reaching the same order of magnitude as the execution time. The pruned submission time curve shows a much more gentle slope instead. If we extrapolate those curves with linear estimations, the extrapolated submission and execution time lines cross at 1,000 nodes without pruning, and at 1,000,000 nodes with pruning. Thus, pruning the task graph lowers the submission cost enough to be able to scale up to many more GPU-accelerated computing nodes than the size of the heterogeneous cluster we used (144 nodes).

The bottom of Figure 4 contains again the submission times, but also a comparison of the number of submitted tasks in the pruned case, against the total number of tasks unrolled by every node. About half of these submitted tasks are actually executed, the other half are needed to infer MPI communications. The increasing discrepancy between those two curves is the main explanation for the benefit of pruning in terms of submission time as seen in the previous para- 
graph. The pruned submission time however increases a bit faster than the number of submitted tasks. The difference is due to the cost of unrolling the loop iterations themselves. Further work is currently in progress, but out of scope of this article, to specify the data mapping at compilation time and use a polyhedral analysis [42] to greatly prune the loop iterations. This could lead to even better scalability while keeping an STF formulation unchanged and thus still very close to Algorithm 7.

\subsubsection{Communication Cache: Limiting Communications and Memory Footprint}

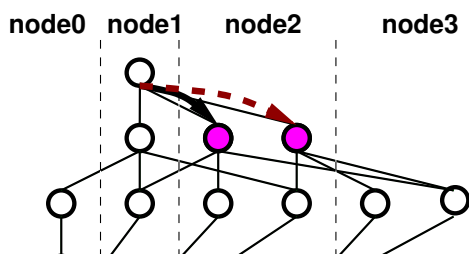

Fig. 5. An example of duplicated communications.

Our distributed unrolling of the (pruned) task graph on all nodes is a fully distributed process, inherently more scalable than a master-slave model by design. Indeed, instead of using explicit synchronization requests between nodes, inter-node communications are initiated from local, decoupled decisions on each side, upon encountering the node's incoming and outgoing dependence edges during the DAG traversal. However, without additional measures, multiple dependence edges involving the same pair of nodes and the same data could trigger redundant network transfers if the corresponding data has not been modified in-between. Figure 5 shows the typical pattern of such a redundant communication. We thus filter redundant messages out using a communication cache mechanism to keep track of the remote data copies that a node already received and which are still up-to-date. The sequential submission of the task graph ensures that the communication cache system follows the expected sequential consistency. In particular, it invalidates a cache entry whenever a remote task writing to the corresponding data is encountered during the task graph traversal.

Figure 6 shows performance results depending on the cache policy, on 16 nodes of the homogeneous setup. Comparing the 'Cache' and 'No Cache' policies confirms the impact on performance of filtering redundant network transfers out through caching. However, the 'Cache' policy reaches an out-of-memory condition for matrices larger than 80,000 . With the exception of pieces of data written into, the runtime system indeed cannot decide when a cached remote copy can safely be evicted without additional information from the application. All valid copies are therefore kept in cache by default.

Hence, we added a method to the StarPU API to allow the application to notify StarPU when some data can safely be flushed from the communication cache. StarPU will actually flush the corresponding cache entry once all tasks using it and submitted before the flush have been executed. Beyond that point, if StarPU encounters a task

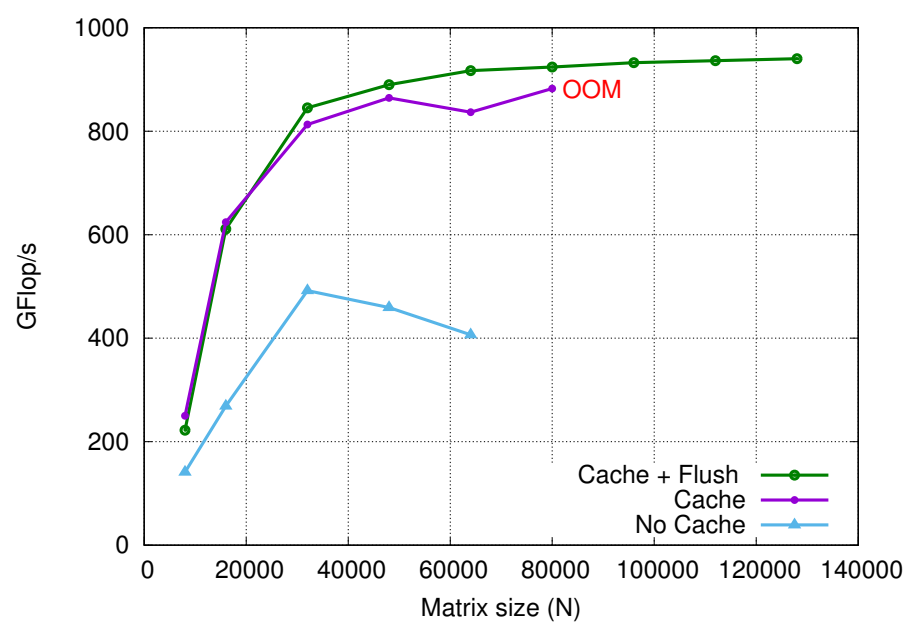

Fig. 6. Impact of communication cache policy on performance.

referencing this data, a new network request will be triggered to get a valid copy. This flush operation can be seen as a notification inserted at some point of the STF submission flow to inform StarPU that the piece of data is possibly not needed by subsequent tasks in a near future. Algorithm 7 shows how this can be added quite naturally within the Cholesky factorization to express, for instance, that the result of a POTRF task is not used beyond the corresponding TRSM tasks. It should be noticed that this is only a memory usage optimization, which does not change any semantics of the computation parts of the task graph. The application developer can thus insert and remove them to improve memory usage without altering the computation correctness. The 'Cache + Flush' policy curve of Figure 6 confirms that matrices of very large scale do not pose outof-memory issues, and that the performance peak can be sustained.

\subsubsection{Runtime-level Allocation Cache}

The allocation of the matrix tiles has to be done carefully. Indeed, depending on the behavior of the system allocator, they would be done along other allocations within the heap, which entails high fragmention issues; or they would be done separately using mmap, whose munmap deallocation is very costly since it requires, for safety, flushing TLB entries on all cores.

We therefore developed and integrated an allocation cache mechanism in StarPU. The mechanism is built on top of mmap in order to be practically immune to fragmentation. It implements pools of reusable memory chunks grouped by size, which drastically reduces the number of expensive calls to mmap and munmap by recycling memory areas from completed tasks - StarPU internal data structures, user data, flushed networking buffers - for newly submitted tasks.

\subsubsection{Controlling the task submission flow}

Submitting tasks as soon as possible enables the runtime system to take decisions early, such as to infer and post the inter-node communications sufficiently ahead of time, to enable (for instance) efficient computation/communication 
overlapping. However, unconstrained bulk submissions of tasks may also lead to unwanted side effects.

The effectiveness of the allocation cache (see Section 4.3.3) is directly dependent on the opportunity, by new tasks being submitted, to reuse memory areas allocated by older tasks that have since gone to completion. Without any constraint on the task submission flow, this reuse opportunity hardly ever happens: the submission time per task is usually much shorter than the execution time (as shown in Figure 4), thus all tasks may already have been submitted by the time opportunities for memory reuse start to arise from task completions.

This problem is emphasized in the case of a distributed session, since StarPU must allocate a buffer for each receive network request posted as the result of submitting a task with an incoming remote dependence. This enables overlapping memory communications with computations. However, this can also lead to the premature allocation of numerous buffers well in advance of their actual usefulness, without additional precautions. The consequences are a larger memory footprint and fewer opportunities for memory reuse due to a larger subset of the buffers being allocated at overlapping times. Here again, the main reason for this issue is that the task submission front usually runs largely ahead of the task execution front.

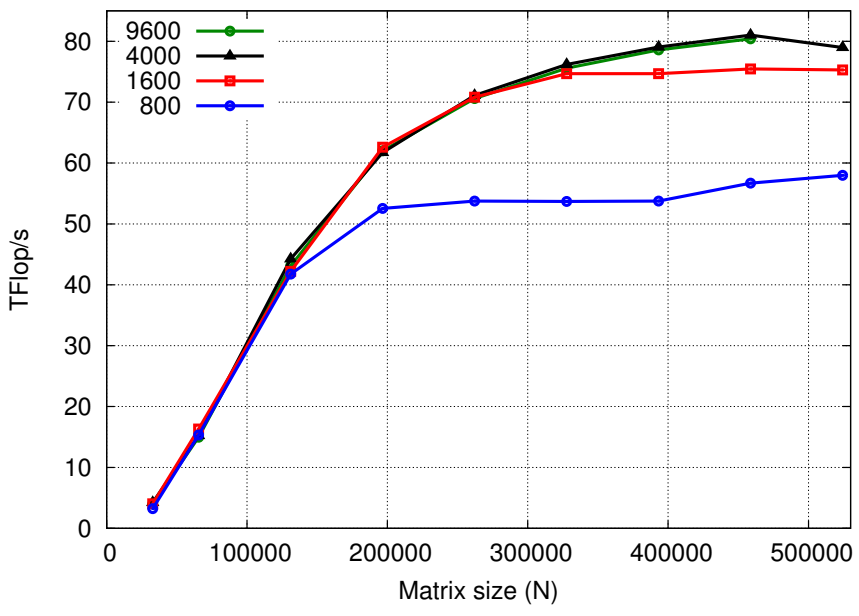

Fig. 7. Performance for several number of tasks thresholds.

A throttling mechanism is therefore necessary on the task submission flow, to keep the memory footprint under control by making the allocation cache effective and by preventing massive, premature allocations of networking buffers. We therefore extended the StarPU API to provide a method for the application to "voluntarily" wait for the number of tasks in the submitted queue to fall below some threshold before continuing its execution. This method can be called for instance at the beginning of an external loop in the application task submission loop nest, to wait for some previously submitted phases to progress, before resuming to submit some new phases. The STF property guarantees that the execution cannot deadlock as the result of task submission being temporarily paused.

For some applications, inserting the voluntarily wait method is not practical or desirable. We hence also provide a similar, but transparent, mechanism at the task submission level inside StarPU. Two environment variables allow to specify an upper and a lower submitted task threshold. When the number of task in the submitted task queue reaches the upper threshold upon a new task submit, the task submission method becomes temporarily blocking. It blocks until the number of remaining tasks in the submitted queue falls below the lower threshold upon which task submission resumes.

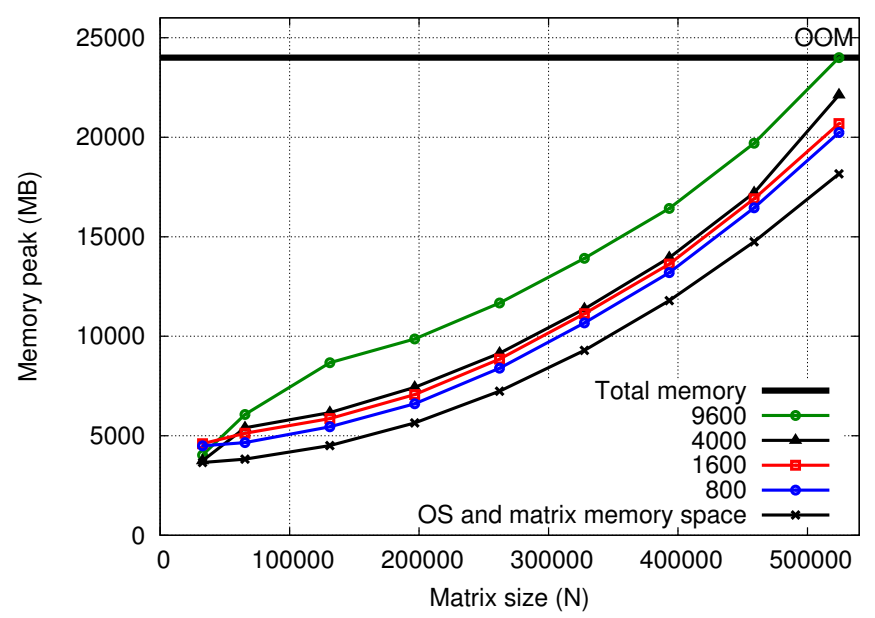

Fig. 8. Memory footprint for several number of tasks thresholds.

Figures 7 and 8 present the performance and memory footprint of our application when the task submission flow is voluntarily controlled at application level, for several choices of submitted task thresholds. Before submitting a new phase of tasks, our application waits until the number of tasks remaining in the submission queue falls below that threshold. Both figures show that a classical trade-off has to be made between available parallelism and lookahead depth on the one side, and memory footprint on the other side, when choosing the value of the threshold. Indeed, we observe that runs using a low task threshold result in lower memory footprint but perform worse due to the more limited available parallelism. Conversely, runs using a high task threshold perform better but have a larger memory footprint, due to the additional networking buffers to allocate simultaneously for an increased number of pending incoming requests.

We explored other criteria for the task submission throttling. One of them is a memory footprint criterion, which temporarily pauses task submission when the amount of memory in use reaches the available size of the system. This ensures that applications with datasets larger than the available memory on the machine may still complete successfully. This was implemented in the StarPU runtime system, and experimented as further work [43], notably using an application with unpredictable memory footprint.

\subsection{Additional test case}

To complement the study performed on the Cholesky factorization, we here present weak scalability results of a 3D regular stencil application implemented with the STF paradigm with a simple block distribution. 160 iterations 
of the stencil were run, and each MPI node had 256 tasks to complete for each iteration. The resulting efficiency is shown on Figure 9. Communications incur about 1.5\% efficiency decrease compared to execution on a single node, but this remains very stable up to the tested 256 nodes of the Occigen cluster. The communications are indeed mostly between neighbors, and thus can scale very well. The task pruning also works extremely well in this case, and a polyhedral analysis easily prunes the spurious loop iterations entirely. Choosing the task number thresholds was also very easy, since they basically correspond to a given number of iterations to be pipelined.

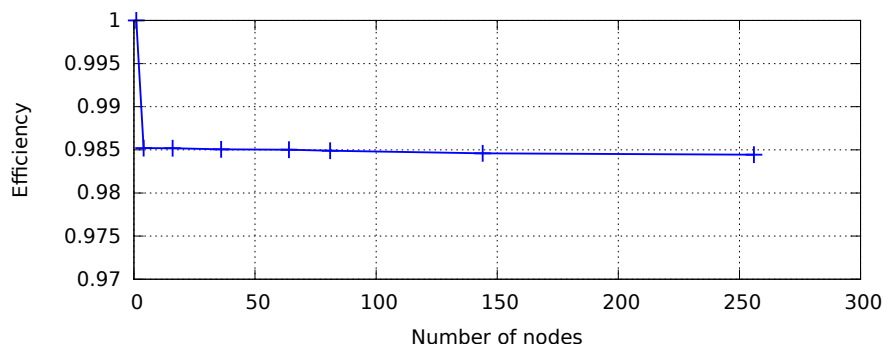

Fig. 9. Efficiency of a stencil application.

\section{Conclusion AND PERSPECtives}

Distributed memory machines composed of multicore nodes possibly enhanced with accelerators have long been programmed with relatively complex paradigms for efficiently exploiting all their resources. Nowadays, most of the HPC applications indeed post communication requests explicitly (MPI paradigm) and their most advanced versions even rely on hybrid programming paradigms in order to better cope with multicore chips (such as MPI+OpenMP) and possibly exploit their accelerators (MPI+OpenMP+Cuda). Task-based paradigms aim at alleviating the programming burden of managing the complex interactions and overlapping between the corresponding runtime systems. The PTG paradigm was designed to achieve a high scalability. It encodes a DAG of tasks as a data flow. However it requires one to explicitly encode the dependencies between tasks. The STF model removes the programming burden of explicitly posting the communications or explicitly providing the dependencies as it infers them automatically. [13] proposed a support of the STF model on homogeneous clusters using a master-slave model. To achieve a higher scalability, YarKhan [24] proposed to unroll the DAG concurrently on all processing units. Yet, the conclusion of the thesis was that "since [PaRSEC, which implements the PTG model] avoids the overheads implied by the [task] insertion [...] it achieves better performance and scalability [than the proposed extension of Quark, which supports the STF model]". In our study, we showed that the STF model can actually compete with the PTG model and we presented the key points that need to be implemented within a runtime system that supports this model to ensure a competitive scalability. Furthermore, our resulting model fully abstracts the architecture and can run any task on any computational unit, making it possible to devise advanced scheduling policies that can strongly enhance the overall performance on heterogeneous architectures. All in all, we could achieve very high performance on a heterogeneous supercomputer while preserving the fundamental property of the model that a unique sequence of tasks is submitted on every node by the application code, to be asynchronously executed in parallel on homogeneous and heterogeneous clusters. To prove this claim, we have extended the StarPU runtime system with an advanced inter-node data management layer that supports this model by automatically posting communication requests. Thanks to this mechanism, an existing StarPU STF application can be extended to work on clusters merely by annotating data with node location to provide the initial data distribution, independently from the algorithm itself. From this data distribution, StarPU infers both the task distribution, along the principle that tasks run on the node owning data they write to, and the inter-node data dependencies, transparently triggering non-blocking MPI_Isend/MPI_Irecv as needed.

We discussed how our design choices and techniques ensure the scalability of the STF model on distributed memory machines. Following [24], we made the choice of a fully decentralized design, made possible by the STF paradigm: every node-local scheduler receives the same task flow from the application, and thus gets a coherent view of the global distributed state, without having to exchange explicit synchronization messages with other nodes. While submitting the whole graph on every node could raise concerns about the scalability [24], we showed that the flow of tasks actually submitted on a given node can be drastically simplified to its distance-1 neighborhood, constituted from the tasks having a direct incoming and/or outgoing dependence with the tasks of this given node. We furthermore implemented two cache mechanisms to offset the expensive cost of memory allocations and avoid redundant data transfer requests, namely the allocation cache and the communication cache respectively. Finally, we designed a throttling mechanism on task submission flow, to monitor resource subscription generated by the queued tasks, and to cap the task submission rate in accordance. Combining the StarPU-MPI layer with these optimizations achieved high performance with the Cholesky factorization on a heterogeneous supercomputer. We showed our approach to be competitive with the state-of-art DPLASMA and ScaLAPACK libraries. All the software elements introduced in this paper are available as part of the StarPU runtime system and the Chameleon dense linear algebra libraries.

On-going and future work on the distributed STF support in StarPU will mainly focus on extending the automation capabilities, on integrating a distributed load balancing engine and generalizing the StarPU-MPI layer networking support. We intend to extend the automation capabilities of StarPU to provide the application programmer with sensible auto-determined thresholds, in particular for the task submission capping mechanism. Since the choice of this parameter value is a trade-off between parallelism and memory footprint, we would like to monitor the memory footprint of tasks, so that tasks can be submitted until all the memory allowed to be used by the runtime system is subscribed. We plan to integrate a load balancing mechanism to alter the initial data distribution automatically 
during the execution to even out the dynamically observed computational load imbalance on every node, relieving the application from that burden. Regarding modern multicore architectures such as Occigen, we are extending StarPU schedulers to better take into account locality and improve per-node performance. We are porting the abstract part of StarPU distributed support on new networking interfaces beyond MPI.

While the STF model has been supported in the OpenMP standard since the 4.0 revision for shared-memory machines with the introduction of the depend clause, we expect that the present work will open up new perspectives for OpenMP towards the support of distributed memory machines.

\section{ACKNOWLEDGMENTS}

This work was done with the support of ANR SOLHAR (grant ANR-13-MONU-0007) as well as the PlaFRIM, Curie/CCRT, and CINES computing centers for the large amount of CPU.hours needed for the large-scale experiments. The authors furthermore thank Cédric Augonnet, David Goudin and Xavier Lacoste from CEA CESTA who strongly contributed to this achievement as well as Luc Giraud and Emmanuel Jeannot for their feedback on a preliminary version of the manuscript.

\section{REFERENCES}

[1] A. Buttari, J. Langou, J. Kurzak, and J. Dongarra, "A class of parallel tiled linear algebra algorithms for multicore architectures," Parallel Computing, vol. 35, pp. 38-53, 2009.

[2] G. Bosilca, A. Bouteiller, A. Danalis, T. Herault, J. Kurzak, P. Luszczek, S. Tomov, and J. Dongarra, "Scalable Dense Linear Algebra on Heterogeneous Hardware," HPC: Transition Towards Exascale Processing, in the series Advances in Parallel Computing, vol. 28, pp. 65-103, 2013.

[3] J. A. Gunnels, F. G. Gustavson, G. M. Henry, and R. A. Van De Geijn, "FLAME: Formal linear algebra methods environment," ACM Transactions on Mathematical Software (TOMS), vol. 27, no. 4, pp. 422-455, 2001.

[4] E. Agullo, C. Augonnet, J. Dongarra, H. Ltaief, R. Namyst, S. Thibault, and S. Tomov, "A Hybridization Methodology for High-Performance Linear Algebra Software for GPUs," in GPU Computing Gems, Jade Edition, vol. 2, pp. 473-484, 2011.

[5] X. Lacoste, M. Faverge, P. Ramet, S. Thibault, and G. Bosilca, “Taking Advantage of Hybrid Systems for Sparse Direct Solvers via Task-Based Runtimes," in Parallel Distributed Processing Symposium Workshops (IPDPSW), 2014 IEEE International. Phoenix, United States: IEEE, May 2014, pp. 29-38.

[6] E. Agullo, A. Buttari, A. Guermouche, and F. Lopez, "Implementing multifrontal sparse solvers for multicore architectures with Sequential Task Flow runtime systems," IRIT, Tech. Rep. IRI/RT-2014-03-FR, November 2014, to appear in ACM Transactions On Mathematical Software. [Online]. Available: http:/ / buttari.perso.enseeiht.fr/stuff/IRI-RT--2014-03--FR.pdf

[7] E. Agullo, L. Giraud, A. Guermouche, S. Nakov, and J. Roman, "Task-based Conjugate Gradient: from multi-GPU towards heterogeneous architectures," Inria, Tech. Rep., 2016.

[8] H. Ltaief and R. Yokota, "Data-Driven Execution of Fast Multipole Methods," Computing Research Repository, vol. abs/1203.0889, 2012.

[9] E. Agullo, B. Bramas, O. Coulaud, E. Darve, M. Messner, and T. Takahashi, "Task-based FMM for multicore architectures," SIAM Journal on Scientific Computing, vol. 36, no. 1, pp. C66-C93, 2014.

[10] B. Lizé, “Résolution directe rapide pour les eléments finis de frontiere en electromagnétisme et acoustique: $\mathcal{H}$-matrices. Parallélisme et applications industrielles," Ph.D. dissertation, Univ. Paris 13, 2014.

[11] T. Heller, H. Kaiser, and K. Iglberger, "Application of the ParalleX Execution Model to Stencil-based Problems," Computer Science Research and Development, vol. 28, no. 2-3, pp. 253-261, 2013.
[12] L. Boillot, G. Bosilca, E. Agullo, and H. Calandra, "Task-based programming for Seismic Imaging: Preliminary Results," in 2014 IEEE International Conference on High Performance Computing and Communications (HPCC). Paris, France: IEEE, Aug. 2014, pp. 12591266.

[13] E. Tejedor, M. Farreras, D. Grove, R. M. Badia, G. Almasi, and J. Labarta, "A high-productivity task-based programming model for clusters," Concurrency and Computation: Practice and Experience, vol. 24, no. 18, pp. 2421-2448, 2012.

[14] J. Bueno, X. Martorell, R. M. Badia, E. Ayguadé, and J. Labarta, "Implementing OmpSs Support for Regions of Data in Architectures with Multiple Address Spaces," in International conference on Supercomputing, 2013, pp. 359-368.

[15] A. YarKhan, J. Kurzak, and J. Dongarra, “Quark users' guide: Queueing and runtime for kernels," University of Tennessee Innovative Computing Laboratory Technical Report ICL-UT-11-02, Tech. Rep., 2011.

[16] A. Zafari, M. Tillenius, and E. Larsson, "Programming models based on data versioning for dependency-aware task-based parallelisation," in International Conference on Computational Science and Engineering, 2012, pp. 275-280.

[17] M. Bauer, S. Treichler, E. Slaughter, and A. Aiken, "Legion: Expressing Locality and Independence with Logical Regions," in International conference on high performance computing, networking, storage and analysis, 2012, pp. 66:1-66:11.

[18] E. Anderson, Z. Bai, J. Dongarra, A. Greenbaum, A. McKenney, J. Du Croz, S. Hammerling, J. Demmel, C. Bischof, and D. Sorensen, "LAPACK: a portable linear algebra library for highperformance computers," in The 1990 ACM/IEEE conference on Supercomputing, 1990, pp. 2-11.

[19] C. Augonnet, S. Thibault, R. Namyst, and P.-A. Wacrenier, "StarPU: A Unified Platform for Task Scheduling on Heterogeneous Multicore Architectures," Concurrency and Computation: Practice and Experience, vol. 23, pp. 187-198, Feb. 2011.

[20] C. Augonnet, J. Clet-Ortega, S. Thibault, and R. Namyst, "DataAware Task Scheduling on Multi-Accelerator based Platforms," in The 16th International Conference on Parallel and Distributed Systems (ICPADS), Shanghai, China, Dec. 2010, pp. 291-298.

[21] M. Cosnard and M. Loi, "Automatic task graph generation techniques," in System Sciences, 1995. Proceedings of the Twenty-Eighth Hawaii International Conference on, vol. 2. IEEE, 1995, pp. 113-122.

[22] G. Bosilca, A. Bouteiller, A. Danalis, M. Faverge, A. Haidar, T. Hérault, J. Kurzak, J. Langou, P. Lemarinier, H. Ltaief, P. Luszczek, A. Yarkhan, and J. J. Dongarra, “Distributed Dense Numerical Linear Algebra Algorithms on massively parallel architectures: DPLASMA," in Proceedings of the 25th IEEE International Symposium on Parallel \& Distributed Processing Workshops and Phd Forum (IPDPSW'11), PDSEC 2011, Anchorage, United States, May 2011, pp. 1432-1441.

[23] A. J. Bernstein, "Analysis of Programs for Parallel Processing," IEEE Transactions on Electronic Computers, pp. 757-763, Oct. 1966.

[24] A. YarKhan, "Dynamic Task Execution on Shared and Distributed Memory Architectures," Ph.D. dissertation, University of Tennessee, 2012.

[25] G. Bosilca, A. Bouteiller, A. Danalis, T. Herault, P. Lemarinier, and J. Dongarra, "DAGuE: A generic distributed DAG engine for High Performance Computing ," Parallel Computing, vol. 38, no. 1-2, pp. $37-51,2012$.

[26] M. Tillenius, E. Larsson, E. Lehto, and N. Flyer, "A task parallel implementation of a scattered node stencil-based solver for the shallow water equations," in Swedish Workshop on Multi-Core Computing, 2013.

[27] E. Slaughter, W. Lee, S. Treichler, M. Bauer, and A. Aiken, "Regent: a high-productivity programming language for HPC with logical regions," in International Conference for High Performance Computing, Networking, Storage and Analysis, 2015, pp. 81:1-81:12.

[28] F. Song and J. Dongarra, "A Scalable Framework for Heterogeneous GPU-based Clusters," in Proceedings of the Twenty-fourth Annual ACM Symposium on Parallelism in Algorithms and Architectures, ser. SPAA '12. New York, NY, USA: ACM, 2012, pp. 91-100.

[29] T. D. Hartley, E. Saule, and Ümit V. Çatalyürek, "Improving performance of adaptive component-based dataflow middleware," Parallel Computing, vol. 38, no. 6-7, pp. 289 - 309, 2012.

[30] C.-K. Luk, S. Hong, and H. Kim, "Qilin: exploiting parallelism on heterogeneous multiprocessors with adaptive mapping," in The 42nd Annual IEEE/ACM International Symposium on Microarchitecture, 2009, pp. 45-55. 
[31] T. Gautier, J. V. F. Lima, N. Maillard, and B. Raffin, "Locality-Aware Work Stealing on Multi-CPU and Multi-GPU Architectures," in Workshop on Programmability Issues for Heterogeneous Multicores (MULTIPROG), 2013.

[32] D. Kunzman, "Runtime support for object-based message-driven parallel applications on heterogeneous clusters," Ph.D. dissertation, University of Illinois, 2012.

[33] D. M. Kunzman and L. V. Kalé, "Programming heterogeneous clusters with accelerators using object-based programming," Scientific Programming, 2011.

[34] Y. Zheng, C. Iancu, P. H. Hargrove, S.-J. Min, and K. Yelick, "Extending Unified Parallel C for GPU Computing," in SIAM Conference on Parallel Processing for Scientific Computing (SIAMPP), 2010.

[35] J. Lee, M. T. Tran, T. Odajima, T. Boku, and M. Sato, " An Extension of XcalableMP PGAS Language for Multi-node GPU Clusters ," in HeteroPar, 2011.

[36] C. Augonnet, S. Thibault, and R. Namyst, "Automatic Calibration of Performance Models on Heterogeneous Multicore Architectures," in Proceedings of the International Euro-Par Workshops 2009, HPPC'09, ser. Lecture Notes in Computer Science, vol. 6043. Delft, The Netherlands: Springer, Aug. 2009, pp. 56-65.

[37] H. Topcuoglu, S. Hariri, and M.-Y. Wu, "Performance-effective and low-complexity task scheduling for heterogeneous computing," Parallel and Distributed Systems, IEEE Transactions on, vol. 13, no. 3, pp. 260-274, Mar 2002.

[38] L. S. Blackford, J. Choi, A. Cleary, E. D'Azeuedo, J. Demmel, I. Dhillon, S. Hammarling, G. Henry, A. Petitet, K. Stanley, D. Walker, and R. C. Whaley, ScaLAPACK user's guide. Society for Industrial and Applied Mathematics, 1997.

[39] “Tera-100/Hyb." 2016, http:/ / www.top500.org/system/177460.

[40] "Occigen," 2016, https://www.top500.org/system/178465.

[41] W. Wu, A. Bouteiller, G. Bosilca, M. Faverge, and J. Dongarra, "Hierarchical DAG Scheduling for Hybrid Distributed Systems," in 29th IEEE International Parallel \& Distributed Processing Symposium, Hyderabad, India, May 2015. [Online]. Available: https:/ /hal.inria.fr/hal-01078359

[42] S. Verdoolaege, J. C. Juega, A. Cohen, J. I. Gómez, C. Tenllado, and F. Catthoor, "Polyhedral parallel code generation for CUDA," ACM Trans. Archit. Code Optim., vol. 9, no. 4, pp. 54:1-54:23, Jan. 2013.

[43] M. Sergent, D. Goudin, S. Thibault, and O. Aumage, "Controlling the Memory Subscription of Distributed Applications with a TaskBased Runtime System," in 21st International Workshop on HighLevel Parallel Programming Models and Supportive Environments, Chicago, United States, May 2016.

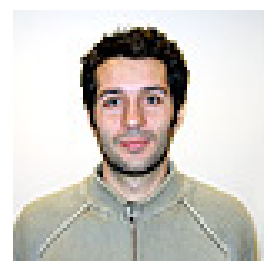

Emmanuel Agullo Dr Emmanuel Agullo holds a permanent Researcher position as part of the HiePACS Inria project team in Bordeaux since 2010. His main research interest is the design of scientific, high performance libraries (especially numerical linear algebra) for modern supercomputers relying on innovative programming paradigms.

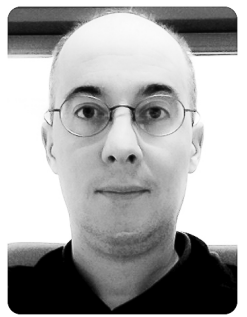

Olivier Aumage Dr Olivier Aumage holds a permanent Researcher position as part of the STORM Team at Inria in Bordeaux since 2003. His research topics include parallel and distributed runtime systems, communication optimization on high performance networks, parallel compilers and performance analysis. He is currently involved in the development of the StarPU runtime, the MAQAO assembly code analyzer and the Klang-omp OpenMP compiler.

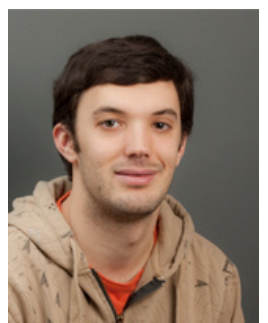

Mathieu Faverge Dr Mathieu Faverge holds an Assistant Professor position at Bordeaux INP and is member of the Inria HiePACS team since 2012. His main research interests are numerical linear algebra algorithms for sparse and dense problems, and especially DAG algorithms relying on dynamic schedulers. He has experience with large distributed heterogeneous systems, and his contributions to the scientific community include efficient linear algebra algorithms for those systems.

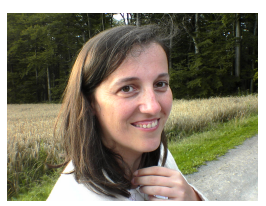

Nathalie Furmento Dr Nathalie Furmento holds a R\&D Engineer Position at the CNRS since 2005. She is part of the Inria STORM team in which she has contributed to the development of several software, the latest being StarPU. Her key interests are high performance distributed computing and runtime systems.

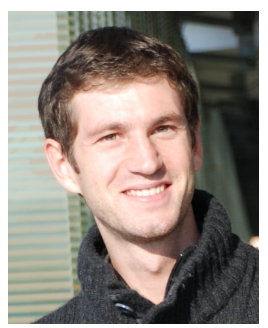

Florent Pruvost Dr Florent Pruvost holds a R\&D contract engineer at Inria since 2013. His research field mainly concerns parallelization techniques for (non-)linear system of equations. He is part of the Inria SED team and is involved in the MORSE project. His work aims at improving the installation and usability of complex software stacks such as linear solvers based on runtime systems, e.g. Chameleon.

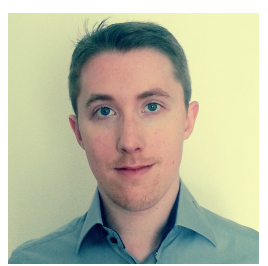

Marc Sergent Marc Sergent received his master's degree in computer science in 2013 from the University of Bordeaux. Since October 2013 he has been working towards a PhD degree as part of the Inria STORM team and the CEA. His research interests focuses on high performance parallel and distributed computing, and particularly task-based programming and runtime systems.

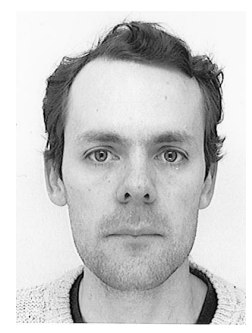

Samuel Thibault Dr Samuel Thibault is Assistant Professor at the University of Bordeaux since 2008, and part of the Inria STORM team. His researches revolve around thread, task, and data transfer scheduling in parallel and distributed runtime systems. He is currently focused on the design of the StarPU runtime, and more particularly its scheduling heuristics for heterogeneous architectures and for distributed systems. 